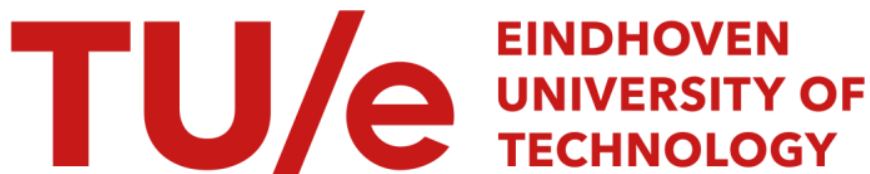

\section{Effectiveness of team training in managing shoulder dystocia}

\section{Citation for published version (APA):}

van de Ven, J., van Deursen, F. J. H. M., van Runnard Heimel, P. J., Mol, B. W. J., \& Oei, S. G. (2016).

Effectiveness of team training in managing shoulder dystocia: a retrospective study. Journal of Maternal-Fetal and Neonatal Medicine, 29(19), 3167-3171. https://doi.org/10.3109/14767058.2015.1118037

DOI:

10.3109/14767058.2015.1118037

Document status and date:

Published: 01/10/2016

\section{Document Version:}

Publisher's PDF, also known as Version of Record (includes final page, issue and volume numbers)

\section{Please check the document version of this publication:}

- A submitted manuscript is the version of the article upon submission and before peer-review. There can be important differences between the submitted version and the official published version of record. People interested in the research are advised to contact the author for the final version of the publication, or visit the $\mathrm{DOI}$ to the publisher's website.

- The final author version and the galley proof are versions of the publication after peer review.

- The final published version features the final layout of the paper including the volume, issue and page numbers.

Link to publication

\section{General rights}

Copyright and moral rights for the publications made accessible in the public portal are retained by the authors and/or other copyright owners and it is a condition of accessing publications that users recognise and abide by the legal requirements associated with these rights.

- Users may download and print one copy of any publication from the public portal for the purpose of private study or research.

- You may not further distribute the material or use it for any profit-making activity or commercial gain

- You may freely distribute the URL identifying the publication in the public portal.

If the publication is distributed under the terms of Article 25fa of the Dutch Copyright Act, indicated by the "Taverne" license above, please follow below link for the End User Agreement:

www.tue.nl/taverne

Take down policy

If you believe that this document breaches copyright please contact us at:

openaccess@tue.nl

providing details and we will investigate your claim. 


\section{Effectiveness of team training in managing shoulder dystocia: a retrospective study}

\section{Joost van de Ven, Frank J. H. M. van Deursen, Pieter J. van Runnard Heimel,} Ben Willem J. Mol \& S. Guid Oei

To cite this article: Joost van de Ven, Frank J. H. M. van Deursen, Pieter J. van Runnard Heimel, Ben Willem J. Mol \& S. Guid Oei (2016) Effectiveness of team training in managing shoulder dystocia: a retrospective study, The Journal of Maternal-Fetal \& Neonatal Medicine, 29:19, 3167-3171, DOI: 10.3109/14767058.2015.1118037

To link to this article: https://doi.org/10.3109/14767058.2015.1118037

册 Published online: 15 Dec 2015.

Submit your article to this journal $\pi$

Џ Article views: 256

Q View related articles $\longleftarrow$

View Crossmark data [ᄌ 


\title{
Effectiveness of team training in managing shoulder dystocia: a retrospective study
}

\author{
Joost van de Ven ${ }^{1}$, Frank J. H. M. van Deursen ${ }^{1}$, Pieter J. van Runnard Heimel ${ }^{1}$, Ben Willem J. Mol ${ }^{1,2}$, and \\ S. Guid Oei ${ }^{1,3}$ \\ ${ }^{1}$ Department of Obstetrics and Gynaecology, Máxima Medical Centre, Veldhoven, The Netherlands, ${ }^{2}$ The Robinson Institute, School of Paediatrics \\ and Reproductive Health, University of Adelaide, Adelaide, Australia, and ${ }^{3}$ Department of Electrical Engineering, Eindhoven University of \\ Technology, Eindhoven, The Netherlands
}

\begin{abstract}
Objectives: To evaluate the effectiveness of simulation team training for the management of shoulder dystocia. Primary outcome measures were the number of reported cases of shoulder dystocia, as well as fetal injury that occurred from it. Secondary outcome is documentation of manoeuvres used to alleviate shoulder dystocia.

Methods: Retrospective cohort study in a teaching hospital in the Netherlands, in a 38 month period before and after implementation of team training.

Results: We compared 3492 term vaginal cephalic deliveries with 3496 deliveries before and after team training. Incidence of shoulder dystocia increased from 51 to 90 cases (RR 1.8 (95\% Cl: $1.3-2.5)$ ). Fetal injury occurred in 16 and eight cases, respectively (RR 0.50 (95\% Cl: 0.21-1.2)). Before team training started, the all-fours manoeuvre was never used, while after team training it was used in 41 of 90 cases (45\%). Proper documentation of all manoeuvres used to alleviate shoulder dystocia significantly increased after team training (RR 1.6 (95\% Cl: 1.05-2.5)).

Conclusions: Simulation team training increased the frequency of shoulder dystocia, facilitated implementation of the all-fours technique, improved documentation of delivery notes and may have a beneficial effect on the number of children injured due to shoulder dystocia.
\end{abstract}

\section{Keywords}

Healthcare team, obstetric labour complications, obstetrics, simulation

\section{History}

Received 20 July 2015

Revised 29 October 2015

Accepted 5 November 2015

Published online 8 December 2015

\section{Introduction}

Shoulder dystocia is an obstetric emergency with serious potential risks for both fetus and mother. Its incidence ranges from $0.2 \%$ to $3 \%$ and it is an uncommon and highly unpredictable event [1,2]. Differences in reported rates seem to reflect significant clinical variation in describing shoulder dystocia. In an effort to establish an objective definition of shoulder dystocia, Spong et al. [3] prospectively studied 250 vaginal deliveries and plotted time intervals for the second stage of labour. Based on their research, this group has proposed that shoulder dystocia should be defined by a prolonged head-to-body delivery time ( $>60 \mathrm{~s}$ ) or the need for ancillary obstetric manoeuvres. It is suggested that poor neonatal outcome after shoulder dystocia is the result of suboptimal management [4,5], in particular excessive traction. Shoulder dystocia increases the neonatal risk of brachial plexus injury, clavicle or humeral fracture, and if not resolved

Address for correspondence: Joost van de Ven, Máxima Medical Centre, Department of Obstetrics and Gynaecology, De Run 4600, 5504 DB Veldhoven, The Netherlands. Tel: +31 408889617. E-mail: j.vandeven1983@gmail.com in a timely fashion, may lead to asphyxia due to umbilical cord compression or even death [6].

Once shoulder dystocia is identified, multiple manoeuvres can be applied in a stepwise fashion in an attempt to alleviate the dystocia: McRoberts manoeuvre, application of suprapubic pressure, rotation of the infant's shoulders and delivery of the posterior arm. Compared with lithotomy position alone, all these manoeuvres reduce both the required delivery force and brachial plexus stretch [7]. An alternative manoeuvre is the all-fours method, also known as the Gaskin manoeuvre, which consists of moving the labouring woman to her hands and knees [8]. Most literature supports McRoberts manoeuvre and suprapubic pressure as reasonable first techniques [9].

Deering et al. [10] reported significant improvements in resident competency in the management of shoulder dystocia after participating in simulation training. Crofts et al. [11] also confirmed the requirement and benefit of simulation training for shoulder dystocia. However, both of these studies consisted of individual simulation training. Our group [12] conducted a systematic review on the effectiveness of multidisciplinary teamwork training in a simulation setting. We reported that obstetric training interventions in a 
simulation setting potentially prevent errors, therefore improving patient safety in acute obstetrics. We also reported in a randomised controlled trial that team performance and medical technical skills improve after team training [13]. Recent literature and experiences in the clinical field show that narrative delivery notes lack many critical elements for documentation of a shoulder dystocia delivery [14].

We hypothesised that simulation team training is an effective method to incorporate a more systematic approach to manage shoulder dystocia including the all-fours technique and to decrease fetal injury as a result of shoulder dystocia.

\section{Methods}

We performed a retrospective cohort study at the Máxima Medical Centre, a teaching hospital in the Netherlands with 1800 deliveries annually. All deliveries are performed by either clinical midwives, resident physicians or gynaecologists. In 2005, simulation team training in acute obstetrics was gradually implemented, using the full body childbirth simulator, Noelle ${ }^{\mathrm{TM}}$ (Gaumard, Miami, FL). The simulation-based team training comprised on average on two scenarios per session. These scenarios consisted of acute obstetric emergencies, including shoulder dystocia. All physicians, clinical midwives and nurses who worked at the delivery rooms were scheduled during working time to attend these training sessions. Team training was given every two weeks by a senior obstetrician and midwife from our centre. When all the care workers were trained the first trainees were trained again in a continuous cycle. During the shoulder dystocia simulation training, technical as well as non-technical skills (e.g. communication) were trained in a simulated delivery room and offered the obstetric providers an opportunity to practice the manoeuvres that can help to alleviate a shoulder dystocia with a minimum amount of force. As the Noelle childbirth simulator is not specifically validated as a training tool for shoulder dystocia, we also used the Prompt Birthing Trainer $^{\mathrm{TM}}$ (Limbs \& Things, Bristol), which monitors traction force in the neck of the baby. During the study period there were no other training interventions.

The management of shoulder dystocia, taught during team training, in our clinic recommends McRoberts manoeuvre, defined as hyper flexion of the maternal legs, as the initial technique. If this manoeuvre is not successful providers then perform suprapubic pressure. Either Rubin's or Woods' corkscrew manoeuvre is subsequently used for rotation of the infant's shoulders and delivery of the posterior arm, when the previous manoeuvres fail to alleviate the shoulder dystocia. When these manoeuvres are unsuccessful the allfours manoeuvre is used, which consists of moving the labouring patient to her hands and knees and gentle traction is applied aiming to deliver the posterior shoulder. During these trainings there was no specific attention given to the comprehensiveness of delivery notes in case of shoulder dystocia.

To assess the effectiveness of the implementation of team training, we analysed in retrospect a 38 month period (from November 2001 to December 2004) before start of the team training and an equal period (from January 2007 to February 2010) after all care workers had been trained.
We included all term $\left(37^{+0}\right.$ to $41^{+6}$ weeks of gestation) vaginal deliveries in cephalic position in the study period. The maternal and neonatal charts of deliveries complicated by shoulder dystocia were reviewed. We also retracted basic data on the periods before and after team training with the use of the Netherlands Perinatal Registry (PRN). These basic data consisted of incidences of diabetic pregnancies, instrumental deliveries, perineal lacerations (divided into second and third/ fourth degree ruptures) and episiotomies. Shoulder dystocia was diagnosed when after delivery of the fetal head, additional obstetric manoeuvres other than gentle downward traction were required. This is a part of the definition proposed by Spong et al. [3]. The prolonged time interval between head and rump was not defined as shoulder dystocia during the team training.

Primary outcome was the number of fetal complications caused by shoulder dystocia, being perinatal asphyxia and neonatal trauma, combined as fetal injury. Asphyxia was described as arterial umbilical cord $\mathrm{pH}<7.05$ and/or Apgar score $<7$ after five minutes, because there is a consistent association with prevalence of neurologic disability, low cognitive function in early adulthood and neonatal mortality $[15,16]$. Neonatal trauma was defined as humeral or clavicle fracture or brachial plexus palsy. Brachial plexus palsy was based on the diagnosis of an attending paediatrician. Fractures were documented by radiographic examination. Neonatal medical charts were reviewed for the diagnosis of fractures or brachial plexus injury. Secondary outcome was documentation in medical charts of all manoeuvres used to alleviate shoulder dystocia.

We compared the outcomes before and after team training using the $\chi^{2}$ test, the Fisher exact test and the calculation of relative risks and $95 \%$ confidence intervals. Ethical approval is not required for this type of study in The Netherlands. SPSS version 18.0 (Chicago, IL) was used to perform statistical analysis.

\section{Results}

There were 6988 term vaginal deliveries in cephalic position with 141 cases of shoulder dystocia during the study period. In the 38 months before obstetric simulation team training started, there were 51 cases $(1.5 \%)$ of shoulder dystocia reported versus 90 cases $(2.6 \%)$ after implementation of team training (RR 1.8 (CI: 1.3-2.5)). In the period after team training there were less instrumental deliveries (RR 0.73 (CI 0.66-0.82)) and less episiotomies (RR 0.89 (CI 0.85-0.94)), but more second-degree ruptures (RR 1.13 (CI 1.05-1.21)) and more gestational diabetes mellitus (GDM) (RR 2.41 (CI 1.23-4.72)). Incidence of third and fourth degree ruptures were alike over the two periods (0.89 (CI 0.66-1.20)) (Table 1).

Among 141 episodes of shoulder dystocia analysed, fetal injury occurred in $24(17.0 \%)$ infants. Injury included two cases $(1.4 \%)$ of brachial palsy, three fractures $(2.1 \%)$ of which one humeral fracture and two fractures of the clavicle bone and 19 cases $(13.5 \%)$ of perinatal asphyxia. Brachial palsy and fracture were present respectively in two and three cases in the period before, and in none of the cases after team training. Perinatal asphyxia related to deliveries complicated 
by shoulder dystocia was reported in 11 cases in the period before and in eight cases in the period after team training was implemented (RR 0.73 (CI: 0.29-1.8)). There were no fetal deaths following shoulder dystocia events during the study period. Before team training, the composite measure of fetal injury occurred in 16 cases versus eight cases after the team training (RR 0.50 (CI: 0.21-1.2)) (Table 2).

In the period before simulation team training, the all-fours manoeuvre was never used, while after implementation the all-fours technique was used in 41 of 90 cases $(45 \%)$ of shoulder dystocia. In 68 cases (48\%) shoulder dystocia was alleviated by initial two techniques: McRoberts manoeuvre and suprapubic impression.

Before team training, in 32 cases of shoulder dystocia all the manoeuvres used were reported, while after team training this was reported in 52 cases (RR 1.6 (CI: 1.05-2.5)). Before

Table 1. Baseline characteristics on period before and after team training.

\begin{tabular}{lccc}
\hline & $\begin{array}{c}\text { After } \\
N=3496\end{array}$ & $\begin{array}{c}\text { Before } \\
N=3492\end{array}$ & $\begin{array}{c}\text { Relative risk } \\
(95 \% \mathrm{CI})\end{array}$ \\
\hline $\begin{array}{l}\text { Gestational diabetes mellitus } \\
\text { Episiotomy }\end{array}$ & 29 & 12 & $2.41(1.23-4.72)$ \\
& 1515 & 1692 & $\begin{array}{c}0.89 \\
(0.85-0.94) \\
0.73\end{array}$ \\
Instrumental delivery & 475 & 648 & $\begin{array}{c}0.66-0.82) \\
1.13\end{array}$ \\
$\begin{array}{l}\text { Perineal laceration } \\
\text { second degree }\end{array}$ & 1119 & 992 & $\begin{array}{c}1.05-1.21) \\
0.89\end{array}$ \\
$\begin{array}{l}\text { Perineal laceration } \\
\text { third/fourth degree }\end{array}$ & 81 & 91 & $(0.66-1.20)$ \\
\hline
\end{tabular}

Table 2. Perinatal outcome in cases complicated by shoulder dystocia.

\begin{tabular}{lccc}
\hline & $\begin{array}{c}\text { After } \\
N=3496\end{array}$ & $\begin{array}{c}\text { Before } \\
N=3492\end{array}$ & $\begin{array}{c}\text { Relative risk } \\
(95 \% \mathrm{CI})\end{array}$ \\
\hline Shoulder dystocia & 90 & 51 & $\begin{array}{c}1.8 \\
(1.3-2.5)\end{array}$ \\
Composite poor perinatal outcome & 8 & 16 & $0.50(0.21-1.2)$ \\
Fracture* & 0 & 3 & Not applicable \\
Brachial palsy* & 0 & 2 & Not applicable \\
Perinatal asphyxia* & 8 & 11 & $0.73(0.29-1.8)$ \\
\hline
\end{tabular}

*In women with shoulder dystocia. implementation of team training, any report on the used manoeuvres was missing in 15 cases, where this was none in the period after team training (Table 3 ).

\section{Discussion}

In this study we found that implementation of team training in acute obstetrics led to an increase of the diagnosis of shoulder dystocia, probably due to a wider definition and personnel at the ward being more alert on documenting the diagnosis and subsequent events. Implementation of the all-fours method was successfully executed and the documentation of the cases of shoulder dystocia improved. There was no significant increase in baseline characteristics in the period after team training that can explain the significant increase in the incidence of cases complicated by shoulder dystocia. Although there is a long list of contributing factors and risk factors that are identified to be associated with shoulder dystocia, many women who have these risk factors will not experience a shoulder dystocia, and many cases of shoulder dystocia occur in the absence of these factors [17].

Team training may have a beneficial effect on the number of children injured as a result of shoulder dystocia. After implementation of team training in none of the cases brachial palsy or a fracture occurred in the newborns. This is possibly a result of the specific shoulder dystocia team training with implementation of the all-fours manoeuvre. During each of the training sessions this manoevre was structurally trained and the Prompt Birthing Trainer ${ }^{\mathrm{TM}}$ (Limbs \& Things, Bristol) was used, which monitors traction force in the neck of the baby. Gurewitsch et al. [18] reported that most clinicians, when asked retrospectively to estimate the amount of traction applied during simulated shoulder dystocia deliveries, underestimate their actual traction by nearly half. This suggests that with rehearsal and real time feedback on traction clinicians will likely be able to increase their skill in using traction in real life situations. All care workers were trained in performing the consecutive steps, including the all-fours manoeuvre, to alleviate shoulder dystocia as described in the Methods section and to limit the amount of force used. There was specific attention to gentle traction on the infant's head; minimum traction force results in less brachial plexus lesions. Crofts et al. [11] reported likewise that care workers who were

Table 3. Manoeuvres used to alleviate shoulder dystocia.

\begin{tabular}{lcccr}
\hline Total cases of shoulder dystocia: 141 & $\begin{array}{c}\text { After team } \\
\text { training }\end{array}$ & $\begin{array}{c}\text { Before team } \\
\text { training }\end{array}$ & $\begin{array}{c}\text { RR } \\
\text { (95\% CI) }\end{array}$ & Total \\
\hline All-fours & 41 & 0 & Not applicable & \\
All manoeuvres reported & 52 & 32 & $1.6(1.05-2.5)$ & 84 \\
McRoberts & 24 & 17 & & 41 \\
McRoberts + suprapubic pressure & 18 & 9 & & 27 \\
McRoberts + suprapubic pressure + internal rotation & 6 & 4 & & 3 \\
McRoberts + internal rotation & 1 & 2 & & 3 \\
McRoberts + all-fours & 3 & 0 & & $4.5(3.4-27)$ \\
One manoeuvre reported & 38 & 4 & & 42 \\
All-fours & 38 & 0 & & 15 \\
Internal rotation & 0 & 2 & & \\
Suprapubic pressure & 0 & 2 & & \\
Manoeuvres not reported & 0 & 15 & Not applicable & 15 \\
\hline
\end{tabular}


trained use less maximal force during alleviation of shoulder dystocia.

In $48 \%$ of the cases of shoulder dystocia management, McRoberts manoeuvre and suprapubic pressure were used. This is in line with international literature which says that these two manoeuvres can alleviate up to $58 \%$ of cases complicated by shoulder dystocia [19]. The incidence of shoulder dystocia in our clinic was $1.5-2.6 \%$ which is in the range of $0.2-3 \%$ as described in actual literature [6]. Incidence of humeral fractures is widely reported from one up to $40 \%$ and in case of clavicle fractures literature reports incidence varying from five to $10 \%$ in cases of shoulder dystocia. One of the largest studies providing comprehensive data on all types of fetal injuries associated with shoulder dystocia indicated that there were $17 \%$ brachial plexus palsies, $10 \%$ clavicle fractures and $4 \%$ humeral fractures [20]. In our study, the incidence of fractures in general, including humeral and clavicle fractures, was half of that (2.1\%). Brachial plexus lesions following shoulder dystocia is seen in $8-18 \%$ of all cases in recent literature. In our study we reported a lower incidence of brachial plexus palsy in case of shoulder dystocia as described earlier. Most previous studies have shown improvements in the management of simulated shoulder dystocia $[10,11]$ and some reported better clinical outcomes $[21,22]$. One of the studies reporting on clinical outcomes is performed by Draycott et al. [21]. This study showed a significant reduction in brachial plexus injury at birth after shoulder dystocia. There was no difference in Apgar scores less than seven at five minutes. Grobman et al. [22] reported recently on the outcomes associated with the introduction of a shoulder dystocia protocol. They showed a significant decrease in brachial plexus palsy, with no difference in perinatal asphyxia. Our study supports the findings of Draycott and Grobman with regard to perinatal asphyxia. Because of the fact that the umbilical $\mathrm{pH}$ decreases on average 0.04 every minute no substantial differences will be seen in the incidence of perinatal asphyxia due to shoulder dystocia. Looking at the overall shoulder dystocia rates Draycott and Grobman both reported similar results in the periods before and after team training where our study describes a 1.8-fold increase in the apparent incidence. In our opinion early recognition and correct alleviation of shoulder dystocia is the key to reduce dystocia-associated neonatal injury. So an increase in total number of shoulder dystocia is justifiable if it results in a decrease of the total numbers of neonatal injury associated with shoulder dystocia. This phenomenon was described in a recent study done in Denmark where more proactive post-term labour induction practice led to an increased risk of shoulder dystocia by $32 \%$, whereas the risk of peripheral nerve injuries was reduced by $43 \%$ [23].

Even though during training sessions there was no specific attention to the comprehensiveness of delivery notes in case of shoulder dystocia, after team training proper report on all manoeuvres used was significantly increased. This is also reported by Grobman et al. [22] where after introduction of a shoulder dystocia protocol a significant improvement in documentation quality was observed. Stohl et al. [14] reported, in $91 \%$ of deliveries complicated by shoulder dystocia, a proper documentation of manoeuvres used to achieve delivery. This implicates a rate of nine per cent of improper documentation. In our study almost 17\% (15 out of 90) of the cases no manoeuvres used to alleviate shoulder dystocia were reported. These deliveries all took place in the period before team training started.

Our study has several limitations including its retrospective design, covering a long period of time and assessment of only medical charts reported by physicians and no nursing reports. Another limitation was that we only analysed data out of our perinatal database of patients who delivered between $37^{+0}$ and $41^{+6}$ weeks of gestation. Patients delivered after $41^{+6}$ weeks are thought to be at a higher risk for shoulder dystocia, unfortunately our data did not specify the mode of delivery for this subgroup. We therefore excluded this group of vaginal deliveries. Reports on GDM are probably underestimated. This incidence was $0.83 \%$ and $0.34 \%$, respectively after and before team training was implemented, where known incidence of GDM in the normal Dutch pregnant population is $3-5 \%$ nowadays [24]. During the study period no screening for GDM in women with known risk factors took place. Only when there were clinical signs (e.g. large for gestational age, polyhydramnion) a glucose tolerance test was performed. Our study consisted of a relative small group of deliveries due to the fact we only analysed data in our own hospital with at that time 1800 deliveries annually. Underpowerment of this study could be the reason that we have found a trend towards less fetal injury due to shoulder dystocia and no statistically significant differences. Satisfaction rates of patients and trained personnel were not assessed. This could be a topic of interest in future prospective clinical research.

\section{Acknowledgements}

The institutional review board of the Máxima Medical Centre judged that ethical approval is not required for this type of study in The Netherlands.

\section{Declaration of interest}

All authors declare that they have no support from any company for the submitted work; their spouses, partners, or children have no financial relationships that may be relevant to the submitted work and they have no non-financial interests that may be relevant to the submitted work.

\section{References}

1. Gross TL, Sokol RJ, Willimas T, Thompson K. Shoulder dystocia: a fetal-physician risk. Am J Obstet Gynecol 1987;156:1408-18.

2. Ouzounian JG, Gherman RB. Shoulder dystocia: are historic risk factors reliable predictors? Am J Obstet Gynecol 2005;192:1933-5.

3. Spong CY, Beal M, Rodriques D, Ross MG. An objective definition of shoulder dystocia: prolonged head-to-body delivery intervals and/or the use of ancillary obstetric maneuvers. Obstet Gynecol 1995;86:433-6.

4. Allen R, Sorab J, Gonik B. Risk factors for shoulder dystocia: an engineering study of clinician-applied forces. Obstet Gynecol 1991; 77:352-5.

5. Hope P, Breslin S, Lamont L, et al. Fatal shoulder dystocia: a review of 56 cases reported to the confidential enquiry into stillbirths and deaths in infancy. Br J Obstet Gynaecol 1998;105: 1256-61.

6. Guidelines of the Dutch society of Obstetrics and Gynecology (NVOG): Schouderdystocie 2008 versie 2.0 (Shoulder dystocia 2008 version 2.0) 
7. Grimm MJ, Costello RE, Gonik B. Effect of clinician-applied maneuvers on brachial plexus stretch during a shoulder dystocia event: investigation using a computer simulation model. Am J Obstet Gynecol 2010;203:339.e1-5.

8. Bruner J, Drummond S, Meenan A, Gaskin I. All-fours maneuver for reducing shoulder dystocia during labor. J Reprod Med 1998;43: 439-43.

9. American College of Obstetricians and Gynecologists. Shoulder dystocia. ACOG Practice Bulletin 40:1-5. Washington (DC): American College of Obstetricians and Gynecologists; 2002.

10. Deering $\mathrm{S}$, Poggi S, Macedonia C, et al. Improving resident competency in the management of shoulder dystocia with simulation training. Obstet Gynecol 2004;103:1224-8.

11. Crofts JF, Bartlett C, Ellis D, et al. Training for shoulder dystocia: a trial of simulation using low-fidelity and high-fidelity mannequins. Obstet Gynecol 2006;108:1477-85.

12. Merién AER, van de Ven J, Mol BW, et al. Multidisciplinary team training in a simulation setting for acute obstetric emergencies: a systematic review. Obstet Gynecol 2010;115:1021-31.

13. Fransen AF, van de Ven J, Merien AE, et al. Effect of obstetric team training on team performance and medical technical skills: a randomised controlled trial. BJOG 2012;119:1387-93.

14. Stohl HE, Granat A, Ouzounian JG, et al. Comprehensiveness of delivery notes for shoulder dystocia. Obstet Gynecol 2014;123: $25 \mathrm{~S}$.

15. Ehrenstein V, Pedersen L, Grijota M, et al. Association of Apgar score at five minutes with long-term neurologic disability and cognitive function in a prevalence study of Danish conscripts. BMC BMC Pregnancy Childbirth 2009;9:14.

16. Malin G, Morris RK, Khan KS. Strength of association between umbilical cord $\mathrm{pH}$ and perinatal and long term outcomes: systematic review and meta-analysis. BMJ 2010;340:c1471

17. Grobman WA. Shoulder dystocia: simulation and a team-centered protocol. Semin Perinatol 2014;38:205-9.

18. Gurewitsch E, Cha S, Johnson T, et al. Traction training for routine and shoulder dystocia delivers: an experimental study. Am J Obstet Gynecol 2005;193:S41.

19. McFarland MB, Langer O, Piper JM, Berkus MD. Perinatal outcome and the type and number of maneuvers in shoulder dystocia. Int J Gynaecol Obstet 1996;55:219-24.

20. Gherman RB, Ouzounian JG, Goodwin TM. Obstetric maneuvers for shoulder dystocia and associated fetal morbidity. Am J Obstet Gynecol 1998;178:1126-30.

21. Draycott TJ, Crofts JF, Ash JP, et al. Improving neonatal outcome through practical shoulder dystocia training. Obstet Gynecol 2008; 112:14-20.

22. Grobman WA, Miller D, Burke C, et al. Outcomes associated with introduction of a shoulder dystocia protocol. Am J Obstet Gynecol 2011;205:513-7.

23. Hedegaard M, Liedegaard O, Skovlund C, et al. Perinatal outcomes following an earlier post-term labour induction policy. BJOG 2015. [Epub ahead of print]. doi: 10.1111/1471-0528.13299.

24. Guideline Diabetes Mellitus and Pregnancy. Version 2.0. Netherlands Society of Obstetrics and Gynecology; 2010. 